\title{
Study of genetic basis of the melanin biosynthesis in barley grain
}

Glagoleva A.Y. ${ }^{*}$, Shmakov N.A. ${ }^{1}$, Mursalimov S.R. ${ }^{1}$, Khlestkina E.K. ${ }^{1,2}$, Shoeva O.Yu. ${ }^{1}$

${ }^{1}$ Institute of Cytology and Genetics, SB RAS, Novosibirsk, Russia

${ }^{2}$ N.I. Vavilov All-Russian Institute of Plant Genetic Resources (VIR), St. Petersburg, Russia

*e-mail:glagoleva@bionet.nsc.ru

In barley (Hordeum vulgare L.), the black grain pigmentation trait caused by the formation of uncharacterized polyphenolic compounds melanins in hulls and pericarp was described. The trait is monogenically inherited by the Blp locus mapped on chromosome $1 \mathrm{H}$. The locus contains 21 genes, nonetheless, the gene controlling black pigmentation has been not identified and isolated yet. The aim of the current study is to carry out comparative analysis of barley near-isogenic lines (NILs) differing by the Blp locus to reveal the most likely candidate gene for Blp. The uncolored cultivar Bowman and its sister line with black grain $\mathrm{i}: \mathrm{Bw} B l p$ were chosen. A comparative morphometric characteristic of the NILs under abiotic stress conditions, as well as a cytological analysis of the developing grain and a transcriptomic analysis in the hulls and pericarp were carried out. Previously the increased resistance to biotic and abiotic stresses of crops with black grain was reported. However, our data demonstrated substantially reduction of the roots and coleoptiles length in the $\mathrm{i}: \mathrm{BwBlp}$ line under drought conditions, but similar response to salinity and heavy metal stress with Bowman. Comparative RNA-seq analysis in the hulls and pericarp of the NILs allowed establishing that the differences in allelic state of the Blp locus are associated with changes in the expression of more than a thousand genes. Among the genes with increased expression in i:BwBlp, the genes of the phenylpropanoids and fatty acids biosynthesis pathways are mostly overrepresented, while the expression of cellulose biosynthesis genes is significantly reduced in this line compared to Bowman. Using light microscopy, the chloroplast localization of melanin pigments was determined.

Acknowledgements: The study was supported by the Russian Science Foundation (No. 16-14-00086). 